\title{
Wave propagation in pulsar magnetospheres
}

\author{
Chen Wang ${ }^{1}$, Dong Lai ${ }^{2}$, Jinlin Han ${ }^{1}$ \\ ${ }^{1}$ National Astronomical Observatories, Chinese Academy of Sciences. A20 Datun Road, \\ Chaoyang District, Beijing 100012, China \\ email: wangchen, hjl@nao.cas.cn \\ ${ }^{2}$ Department of Astronomy, Cornell University, Ithaca, NY 14853, USA \\ email: dong@astro.cornell.edu
}

\begin{abstract}
We study the propagation effects of radio waves in a pulsar magnetosphere, composed of relativistic electron-positron pair plasmas streaming along the magnetic field lines and corotating with the pulsar. We critically examine the various physical effects that can potentially influence the observed wave intensity and polarization. We numerically integrate the transfer equations for wave polarization in the rotating magnetosphere, taking account of all the propagation effects in a self-consistent manner. For typical magnetospheric plasma parameters produced by pair cascade, we find that the observed radio intensity and polarization profiles can be strongly modified by the propagation effects. Some applications of our results are discussed.
\end{abstract}

Keywords. pulsars: general, plasmas, polarization, magnetic fields, radiative transfer

\section{Introduction}

Pulsar radio emission is likely generated within a few hundred kilometers from the neutron star (NS) surface (e.g. Cordes 1978; Blaskiewicz et al. 1991; Kramer et al. 1997; Kijak \& Gil 2003). A pulsar is surrounded by a magnetosphere filled with relativistic electron-positron pair plasmas (plus possibly a small amount of ions) within the light cylinder. When radio waves propagate though the magnetosphere, the total flux, polarization state and spectrum of the emission may be modified by propagation effects.

Wave propagation effects in pulsar magnetopheres play an important role in the diverse behavior of pulsar polarization, such as the origin of circular polarization $(\mathrm{CP})$ and orthogonal polarization modes (OPM) - see, e.g., Lyubarsky 2008 for a review. A number of theoretical works have been devoted to study how magnetosphere propagation influences pulsar polarization observations (Cheng \& Ruderman 1979, Arons \& Barnard 1986, Barnard \& Arons 1986, Lyubarskii \& Petrova 1998, Luo \& Melrose 2001, Petrova 2006). However, none of the previous studies have calculated the final polarization profiles with all of these propagation effects included in a self-consistent way within a single theoretical framework. It is often unclear which of the effects are most important, and if so, under what conditions. In our works (Wang, Lai \& Han 2010, hereafter WLH10), we attempt to combine all the propagation effects, evaluate their relative importance, and use numerical integration along the photon ray to study the influence of propagation effects on the final polarization states.

\section{Some typical propagation effects}

Adiabatic walking (Cheng \& Ruderman 1979). If the plasma properties change very slowly and the mode evolution is adiabatic, the two natural wave modes will propagate independently. For example, an initially O-mode photon will always stay in O-mode, which means its polarization direction follows the magnetic field plane along the ray. 
Wave mode coupling. When a photon propagates through the magnetosphere to outside space, mode evolution will become non-adiabatic at a position called the "polarizationlimiting-radius" $\left(r_{\mathrm{pl}}\right)$. Near the radius, the two natural wave modes will be coupled, CP can be generated. Away from the radius, the polarization state will be frozen and not change again.

Quasi-tangential effect (Wang \& Lai 2009). When the photon crossed the region where its wave vector is aligned or nearly aligned with the magnetic field, the azimuthal angle of the magnetic field changes quickly, and mode coupling may occur. This effect only works for small impact angles.

Circularization. If the wave vector is very close to the magnetic axis, or the pair plasma is asymmetric, the natural wave modes could become circular polarized near the polarization-limiting-radius. Thus, even if the photon is initially $100 \%$ linearly polarized, the final polarization state is determined by the natural wave modes near $r_{\mathrm{pl}}$, which may be partially circularly polarized.

Cyclotron absorption. The emission will be absorbed near the cyclotron resonance where the wave frequency in the plasma rest frame is close to the cyclotron frequency.

\section{Numerical integration of wave propagation in mangetosphere}

In many cases these different propagation effects are coupled and not easy to separate. Thus, to produce the observed polarization profiles, it is necessary to use the numerical ray integrations to calculate the final wave polarization states.

Polarization evolution for a single ray. We choose a typical emission height $r_{\mathrm{em}}=50 R_{*}$ and assume that at the emission point, the photon is polarized in the magnetic field line plane (O-mode). We can calculate the dielectric tensor at each point along the photon ray, and integrate the wave evolution equation (see Eq. 2.10 of WLH10) from the emission point to a large radius (generally close to the light cylinder $r_{\mathrm{lc}}$ ), and get the final polarization state of the photon. It is clear that wave mode coupling and cyclotron absorption are the most obvious effects for all the cases.

Polarization Profiles of Pulsar Emission Beam. Assuming that emission at various pulsar rotation phases are from the same height, we can extend the single ray evolution results to the polarization profiles of pulsar emission beam after the propagation effects in pulsar magnetosphere. Figure 1 shows a typical example of the phase evolution of the intensity and polarization. We find the intensity and polarization profiles are strongly affected by the propagation effects, such as:

1) The intensity profile is modified by phase-different cyclotron absorption, the trailing side being absorbed more.

2) The PA curve is close to RVM only when the impact angle is not so small, and Lorentz factor and plasma density are not so large. There exits a shift away from the RVM predicted curve (Fig. 1a), which is $r_{\mathrm{pl}} / r_{\mathrm{lc}}$.

3) For a large Lorentz factor and plasma density, or very small impact angles, the PA curve is more complicated and deviates far away from the RVM predictions (Fig. 1b-d). In some cases, the observed PA curve has a $90^{\circ}$ jump accompanied by the maximum CP (Fig. 1b), which may be a possible origin of orthogonal polarization modes in some pulsars. The circular polarization curve could have sign reversals in this case.

\section{Conclusions}

We use numerical integration of the photon polarization along the ray to incorporate all these propagation effects in magnetosphere self-consistently within a single frame- 


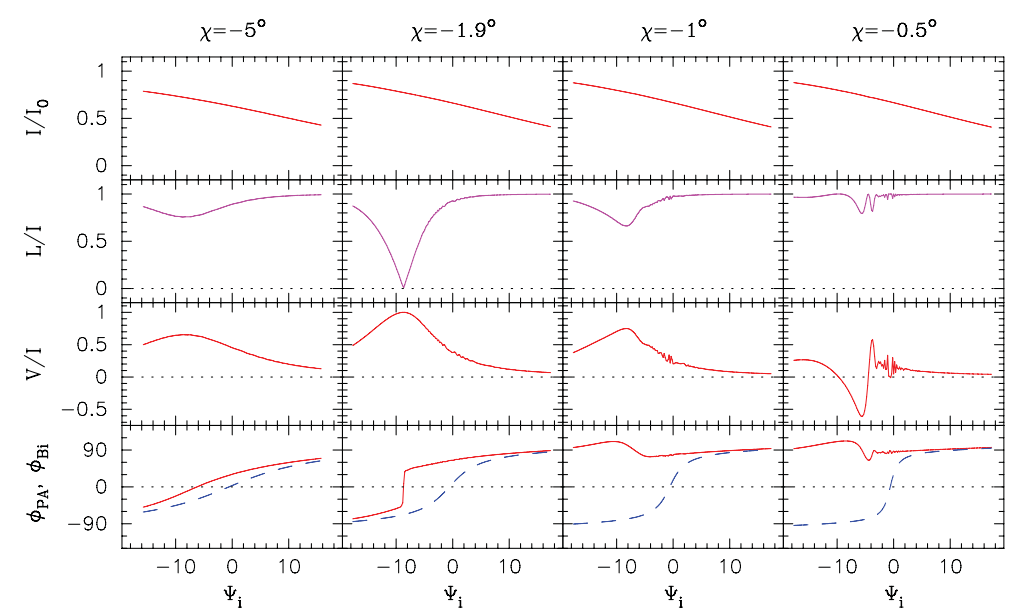

Figure 1. Intensity and polarization profiles of pulsar emission beam. The four columns correspond to four fixed impact angles $\chi=-5^{\circ},-1.9^{\circ},-1^{\circ}$ and $-0.5^{\circ}$, respectively. The horizontal axis $\Psi_{\mathrm{i}}$ is the pulsar rotation phase. In each column, we plot the Stokes parameters $I / I_{0}$ (top panel), $L / I$ (second panel), $V / I$ (third panel), $\phi_{\mathrm{PA}}$ and $\phi_{B \mathrm{i}}$ (bottom panel, the solid line for $\phi_{\mathrm{PA}}$ and the dashed for $\left.\phi_{B \mathrm{i}}\right)$. The initial polarization states are all ordinary mode, and the other parameters are: surface magnetic field $10^{12} \mathrm{G}$, NS spin period $P=1 \mathrm{~s}$, wave frequency $\nu=1 \mathrm{GHz}$, Lorentz factor $\gamma=100$, inclination angle $\alpha=30^{\circ}$, and emission height $r_{\mathrm{em}}=50 R_{*}$.

work. We find that, for typical parameters of the magnetospheric plasma produced by pair cascade, the final intensity and polarization position angle can be strongly modified by the propagation effects. Although we adopted the simplest (and minimum) assumptions about the property of the magnetospheric plasma and the intrinsic radio emission mechanism, our results already show great promise in explaining a number of otherwise puzzling observations, such as the phase-shift of $\mathrm{PA}$ curve, $\mathrm{CP}$ origin, orthogonal mode plarization. An interesting application is that for the so-called conal double type pulsars, which in our model corresponds to large impact angle $\chi$, the relationship between the single sign of the circular polarization and the derivation of $\phi_{\mathrm{PA}}$ (see Han et al. 1998) can be easily understood if $\mathrm{CP}$ is generated by wave mode coupling effect.

\section{References}

Arons, J. \& Barnard, J. J. 1986, ApJ, 302, 120

Barnard, J. J. \& Arons, J. 1986, ApJ, 302, 138

Blaskiewicz, M., Cordes, J. M., \& Wasserman, I. 1991, ApJ, 370, 643

Cheng, A. F. \& Ruderman, M. A. 1979, ApJ, 229, 348

Cordes, J. M. 1978, ApJ, 222, 1006

Han, J. L., Manchester, R. N., Xu, R. X., \& Qiao, G. J. 1998, MNRAS, 300, 373

Kijak, J. \& Gil, J. 2003, A\&ٌA, 397, 969

Kramer, M., Xilouris, K. M., Jessner, A., et al. 1997, A\&SA, 322, 846

Luo, Q. H. \& Melrose, D. B. 2001, MNRAS, 325, 187

Lyubarsky, Y. 2008, in 40 YEARS OF PULSARS, eds. C. Bassa, Z. Wang, A. Cumming, \&

V. M. Kaspi, AIP Conf. Ser., 983, 29

Lyubarskii, Y. E. \& Petrova, S. A., 1998, ApESSS, 262, 379

Petrova, S. A. 2006, MNRAS, 366, 1539

Wang, C. \& Lai, D. 2009, MNRAS, 398, 515

Wang, C., Lai, D., \& Han, J. L. 2010, MNRAS, 403, 569 